\title{
Leveraging machine learning-based approaches to assess human papillomavirus vaccination sentiment trends with Twitter data
}

\author{
Jingcheng Du, Jun Xu, Hsing-Yi Song and Cui Tao*
}

From The International Conference on Intelligent Biology and Medicine (ICIBM) 2016

Houston, Texas, USA. 08-10 December 2016

\begin{abstract}
Background: As one of the serious public health issues, vaccination refusal has been attracting more and more attention, especially for newly approved human papillomavirus (HPV) vaccines. Understanding public opinion towards HPV vaccines, especially concerns on social media, is of significant importance for HPV vaccination promotion.

Methods: In this study, we leveraged a hierarchical machine learning based sentiment analysis system to extract public opinions towards HPV vaccines from Twitter. English tweets containing HPV vaccines-related keywords were collected from November 2, 2015 to March 28, 2016. Manual annotation was done to evaluate the performance of the system on the unannotated tweets corpus. Followed time series analysis was applied to this corpus to track the trends of machine-deduced sentiments and their associations with different days of the week.
\end{abstract}

Results: The evaluation of the unannotated tweets corpus showed that the micro-averaging F scores have reached 0.786. The learning system deduced the sentiment labels for 184,214 tweets in the collected unannotated tweets corpus. Time series analysis identified a coincidence between mainstream outcome and Twitter contents. A weak trend was found for "Negative" tweets that decreased firstly and began to increase later; an opposite trend was identified for "Positive" tweets. Tweets that contain the worries on efficacy for HPV vaccines showed a relative significant decreasing trend. Strong associations were found between some sentiments ("Positive", "Negative", "Negative-Safety" and "Negative-Others") with different days of the week.

Conclusions: Our efforts on sentiment analysis for newly approved HPV vaccines provide us an automatic and instant way to extract public opinion and understand the concerns on Twitter. Our approaches can provide a feedback to public health professionals to monitor online public response, examine the effectiveness of their HPV vaccination promotion strategies and adjust their promotion plans.

Keywords: Sentiment analysis, Human papillomavirus vaccines, Machine learning, Twitter, Hierarchical classification

\footnotetext{
* Correspondence: cui.tao@uth.tmc.edu

University of Texas School of Biomedical Informatics, 7000 Fannin St Suite 600, Houston, TX 77030, USA
} 


\section{Background}

With the rise of social media and the burgeoning volume of user-generated data, there is a growing interest in using social media data for public health related studies. Social media surveillance has proven its value for many public health issues, such as estimating of epidemiological patterns [1], forecasting disease outbreak $[2,3]$, detecting drug adverse effects [4], and assessing vaccination [5, 6]. With 310 million monthly active users and 500 million tweets posting per day [7], Twitter is one of the largest and most popular social media in US and in the world. The length limit of 140 characters per tweet also makes users post more concisely and more expressively than other social networks and blogs [8]. These characteristics make Twitter a very valuable data source for public health informatics researchers.

Vaccination refusal has been a serious issue for human papillomavirus (HPV) vaccines [9]. Introduced in 2006, HPV vaccine can be used to prevent most cancers caused by HPV infections. However, compared to other recommended vaccines, HPV vaccines coverage in USA is still quite low especially among adolescents [10]. Antivaccine rhetoric directed at HPV vaccines in media and online appears to be able to alter vaccine acceptance and decision-making [5]. As individuals' decisions about whether or not to immunize are not usually made rationally nor at one moment in time [11] and individual health behaviors appear to be modulated by opinions from social networks [6], understanding public opinion towards HPV vaccines in social media is of significant importance for HPV vaccination promotion. Moreover, the surveillance of real-time Twitter information flow could provide timely updates when scares arise, and instant feedback to public health agencies to examine and adjust their strategies to improve future HPV vaccines uptake and adherence.

Unlike traditional surveying methods that are laborintensive and expensive [6], we propose to leverage machine learning approaches to extract public opinion from tweets automatically. This is called sentiment analysis (SA) in the field of natural language processing (NLP). In our previous work, we developed a machine learning based sentiment analysis system that can hierarchically classify HPV vaccine-related tweets into 10 categories [12]. This system not only will deduce the sentiment polarity of a tweet at the high level (e.g., "Positive", "Negative" and "Neutral"), but will also further identify the exact reasononing behind a negative opinion (e.g., "Safety", "Efficacy", etc.). In this study, we evaluated this system on a large-scale unannotated tweets corpus and deduce the sentiment labels of those tweets. Additional time series analysis and regression models were applied to track the changes and to identify the patterns of different sentiments toward HPV vaccines over time.

\section{Methods}

\section{Data resource}

We used a set of keywords (hpv, human papillomavirus, gardasil, and cervarix) to collect English tweets by using Twitter Streaming APIs. The tweets corpus was collected from November 2, 2015 to March 28, 2016. There were 184,214 tweets collected during that time period. We will use this corpus to evaluate the sentiment analysis system developed in our previous work and leverage the system to extract and analyze public opinion toward HPV vaccines from the unannotated tweets corpus.

\section{Machine learning system}

A machine learning based sentiment analysis system was developed in our previous work to extract public opinions from HPV vaccines related tweets [12]. This system was able to classify tweets into multiple sentiment categories. Figure 1 shows the sentiment classification scheme for the HPV vaccines related tweets. Detailed definitions of each category were provided in Additional file 1. By leveraging hierarchical classification methods (three SVM models) with optimized feature sets and model parameters, this system has achieved the microaveraging F score at 0.7442 on the gold standard [12]. The overview of the sentiment analysis system can be seen in Fig. 2.

\section{Evaluation of the system on the unannotated tweets corpus}

To evaluate system performance on the unannotated tweets corpus, we randomly selected 500 tweets from the unannotated tweets corpus and manually annotated those tweets according to the sentiment classification scheme. Then, the machine learning system was applied to deduce the sentiment labels of these annotated tweets. Machine learning system-deduced sentiment labels were compared with manually annotated labels. Standard metrics including precision, recall and F measure

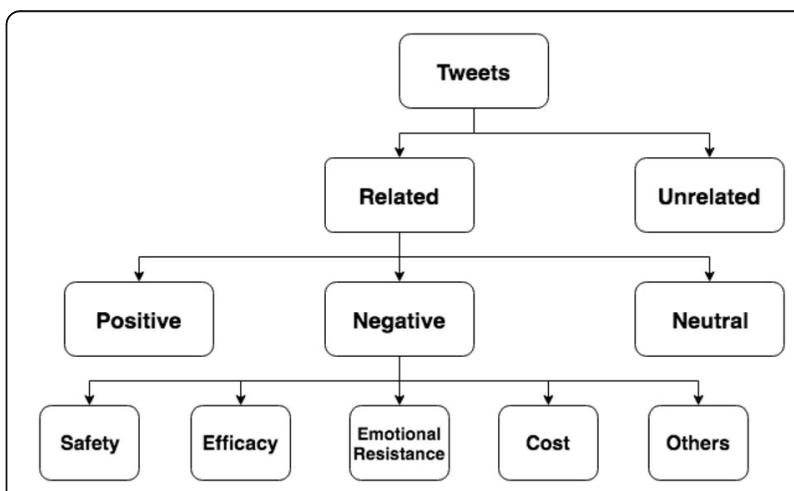

Fig. 1 Sentiment classification scheme for HPV vaccine related tweets [12] 


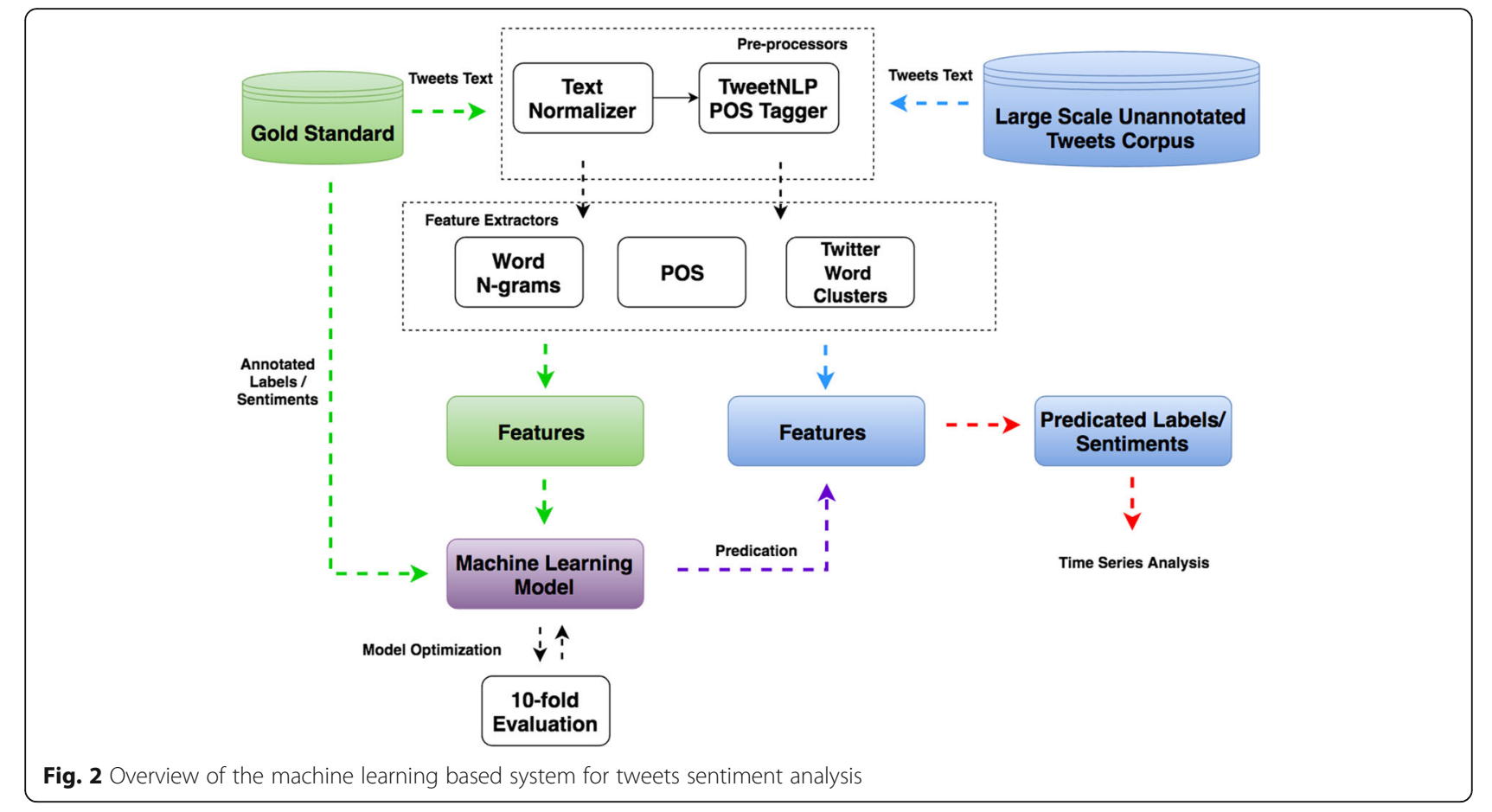

were then calculated to evaluate the performance of the system. We also calculated the micro-averaged and macro-averaged score to evaluate the overall performance on all sentiment categories. To calculate the microaveraged score, we summed up all the individual true positives, false positives, and false negatives. For the macroaveraged score, we calculated the average score of the $\mathrm{F}$ measure of all sentiment categories.

\section{Time series analysis on predicated sentiments}

After the evaluation, we leveraged our system to deduce the sentiment labels for all the tweets in the unannotated tweets corpus. Longitudinal deduced counts and rates for different sentiment categories were calculated. Time series trends for different sentiment categories were graphed and analyzed by Tableau. As social media posting behavior is associated with different days of the week [13, 14], we also calculated the averaged rates of different sentiment groups for different days of the week. Linear and quadratic regression models were used to fit the data to explore trends and associations.

\section{Results}

\section{Machine learning system evaluation results}

Among the 500 randomly selected tweets from the large corpus, our manual annotation found that 193 of them were "Unrelated" to HPV vaccine sentiments, 116 tweets were "Neutral", and 106 tweets were "Positive". There were also 65 tweets and 20 tweets that were annotated into "NegSafety" and "NegOthers" sentiment categories, respectively. The micro-averaged and macro-averaged of F scores have reached 0.786 and 0.708 respectively. The overall performance was promising. The detailed evaluation results for the overall performance and for each category can be seen in Table 1 .

\section{Overall description of the predicated sentiments}

Our machine learning system then deduced the sentiment labels for 184,214 tweets in the large unannotated tweets corpus from the study period. Overall, 110,778 (60.13\%) tweets were classified into the "Related" group. Among the related tweets, 39,704 (35.8\%) of them showed positive opinions; 35,591 (32.1\%) tweets are categorized into "Neutral"; and 35,482 (32.0\%) tweets were categorized into "Negative". The largest group under the "Negative" tweets is "NegSafety". There were 28,108 tweets classified into this category. Besides, 7252 tweets

Table 1 Machine learning system evaluation on 500 randomly selected tweets from the unannotated tweets corpus

\begin{tabular}{|c|c|c|c|c|c|c|}
\hline \multicolumn{4}{|l|}{ Category } & \multirow{2}{*}{$\begin{array}{c}\text { Precision } \\
0.7860\end{array}$} & \multirow{2}{*}{$\begin{array}{l}\text { Recall } \\
0.7860\end{array}$} & \multirow{2}{*}{$\begin{array}{c}\text { F measure } \\
0.7860\end{array}$} \\
\hline \multirow[t]{2}{*}{ Overall } & \multicolumn{3}{|c|}{ Micro-averaging } & & & \\
\hline & \multicolumn{3}{|c|}{ Macro-averaging } & 0.7112 & 0.7051 & 0.7081 \\
\hline \multirow[t]{5}{*}{ Per Category } & \multicolumn{3}{|c|}{ Unrelated } & 0.9337 & 0.9482 & 0.9409 \\
\hline & \multirow[t]{4}{*}{ Related } & Positive & & 0.6596 & 0.8774 & 0.7530 \\
\hline & & Neutral & & 0.7586 & 0.5690 & 0.6502 \\
\hline & & Negative & Safety & 0.8039 & 0.6308 & 0.7069 \\
\hline & & & Others & 0.4000 & 0.5000 & 0.4444 \\
\hline
\end{tabular}


and 123 tweets were categorized into "NegOthers" and "NegEfficacy" respectively. No tweets were classified into "NegCost" or "NegResistant". Detailed sentiments distribution can be seen in Fig. 3. Sample tweets predicated by the machine learning system for different sentiment categories were provided in the Additional file 2.

\section{Time series analysis for different sentiment categories}

Figure 4 presents an overview of the changing number of different sentiment tweets from November 2, 2015 to March 28, 2016. The average posts for all categories were 1245 per day. A sharp peak was found on Feb 22, 2016. This peak was coincided with an article on The New York Times titled "HPV Sharply Reduced in Teenage Girls Following Vaccine, Study Says" [15]. This article was published exactly on Feb 22, 2016. This coincidence showed an interaction between mainstream events and Twitter contents.

We calculated the relative daily proportion of "Positive", "Negative" and "Neutral" tweets to "Related" tweets respectively. Figure 5 shows the time series of different sentiments proportion from November 2, 2015 to March 28, 2016. One of the peaks for "Positive" was found around Feb 22, 2016 (at 66.21\%), when The New York Times published the aforementioned article. It can be used as an example to show how real-world media can influence the HPV vaccines public opinion on social media.

Quadratic models were then used to fit the time series data to explore trends. We can observe that there were strong fluctuations of the relative proportion for these three sentiment groups. For the "Negative" group, we can

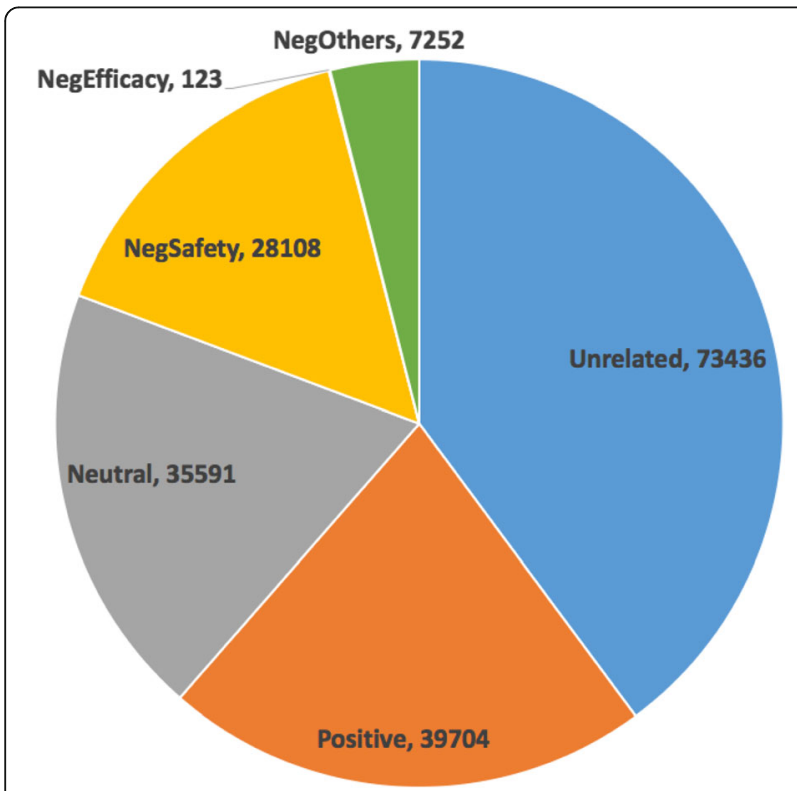

Fig. 3 Sentiments distribution in large scale unannotated HPV vaccines related tweets corpus. (Neg: Negative) see a weak trend that the relative proportion decreased firstly and began to increase around Feb, 2016; for the "Positive" group, we can observe a trend opposite to the "Negative" group, which increased at first and started to decrease around Feb, 2016; for "Neutral" tweets, the curve of the fitted quadratic model was relatively flat.

To explore the time series trend of the sub-category of "Negative", we further calculated the relative daily proportion of "NegSafety", "NegEfficacy" and "NegOthers" tweets to "Negative" tweets respectively, see Fig. 6, Two main peaks have been found for "NegOthers" sentiment group: $72.92 \%$ on Jan 4, 2016 and $80.95 \%$ on Mar 2, 2016. Linear models were fitted to explore the trends. We can observe that there was relative significant decreasing trend for "NegEfficacy" sentiment group. For "NegSafety" and "NegOthers" groups, no significant trends were identified.

In order to explore the association of people's posting behavior and sentiments with different days of the week, we went one step further and calculated the average rates of different sentiment groups on different days of the week. We applied the quadratic models to fit the data. Figure 7 shows the association of "Negative", "Neutral" and "Positive" sentiment groups with different days of the week. For the "Negative" sentiment group, we found that the quadratic models fit the data quite well $\left(R^{2}=0.992\right)$. The average rate for "Negative" came to the bottom (around 34\%) on Wednesdays and reached the peak on weekends. For "Positive" tweets, the quadratic model also fitted the trends quite well $\left(R^{2}=0.992\right)$. The trend for "Positive" tweets is quite opposite to "Negative" tweets. The average rate for "Positive" reached the peak at the middle of a week and came to the bottom on the weekends. No significant association was found for the "Neutral" sentiment group.

The association of "NegSafety", "NegEfficacy", and "NegOthers" tweets with different days of the week can be seen in Fig. 8. The quadratic models fitted the "NegSafety" and "NegOthers" sentiment groups quite well ( $R^{2}$ at 0.876 and 0.917 respectively). The relative proportion for "NegSafety" reached the bottom (around 76\%) on Wednesdays and peaked on weekends. For "NegOthers" tweets, the average rate reached the peak at the middle of the week and was lowest on the weekends. No significant association was found for the "NegEfficacy" sentiment group.

\section{Discussion}

Our analysis found the coincidence between mainstream events (The New York Times article) and Twitter contents. We also found that the activity on mainstream media can have a significant influence on HPV vaccine public opinion on Twitter (with $66.21 \%$ positive rate on the article posting day compared to $35.8 \%$ positive rate 

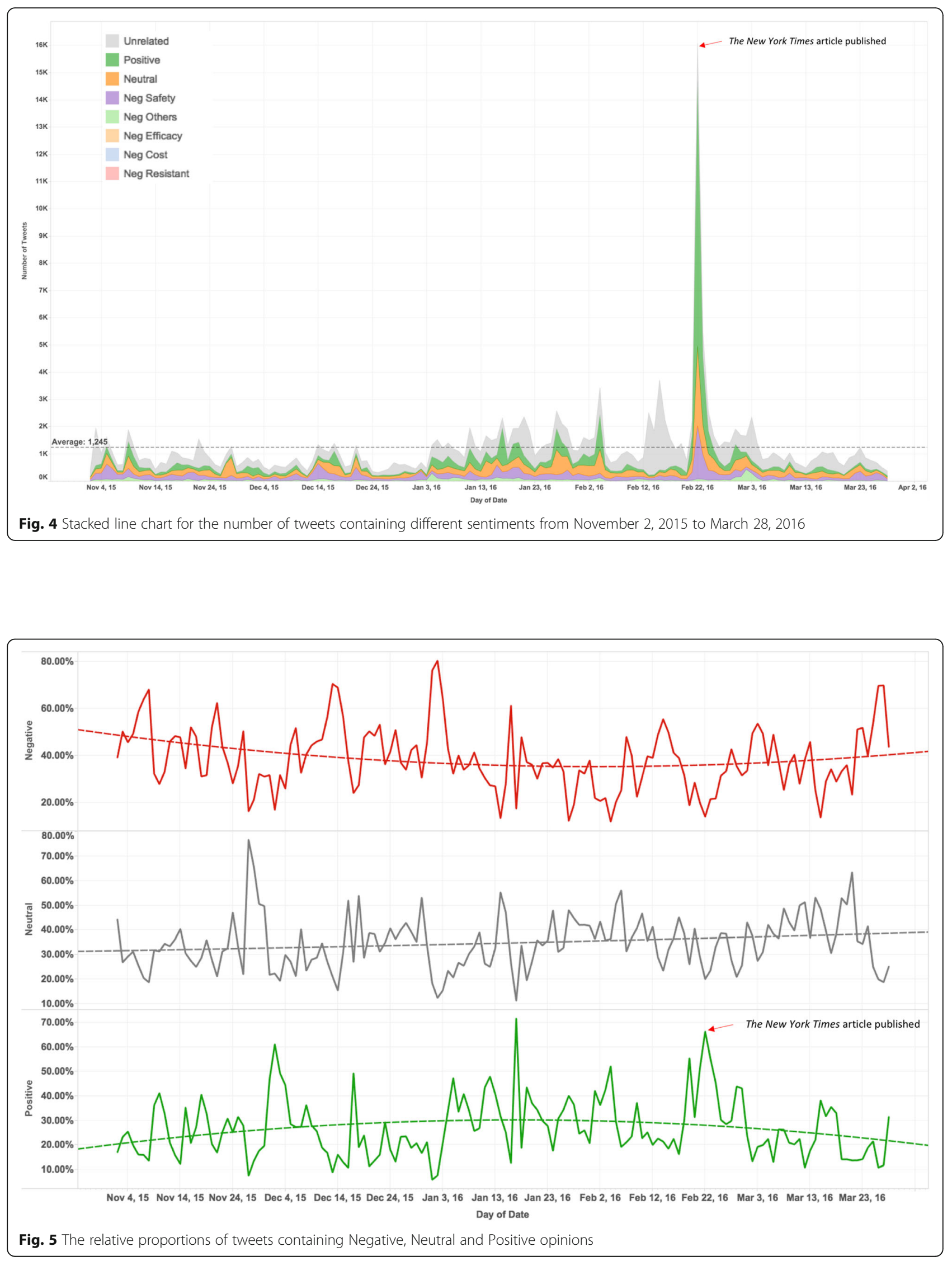


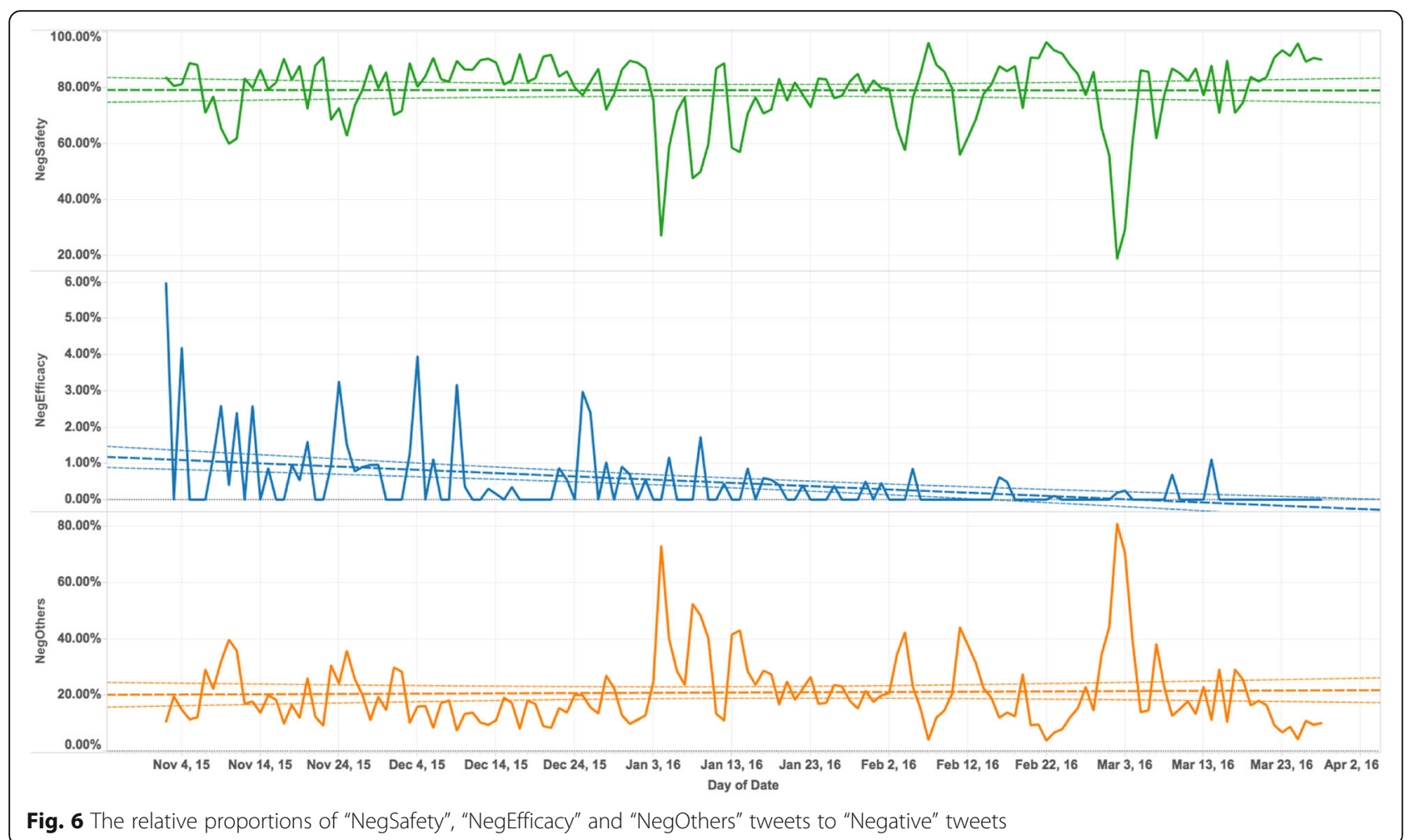

Fig. 6 The relative proportions of "NegSafety", "NegEfficacy" and "NegOthers" tweets to "Negative" tweets

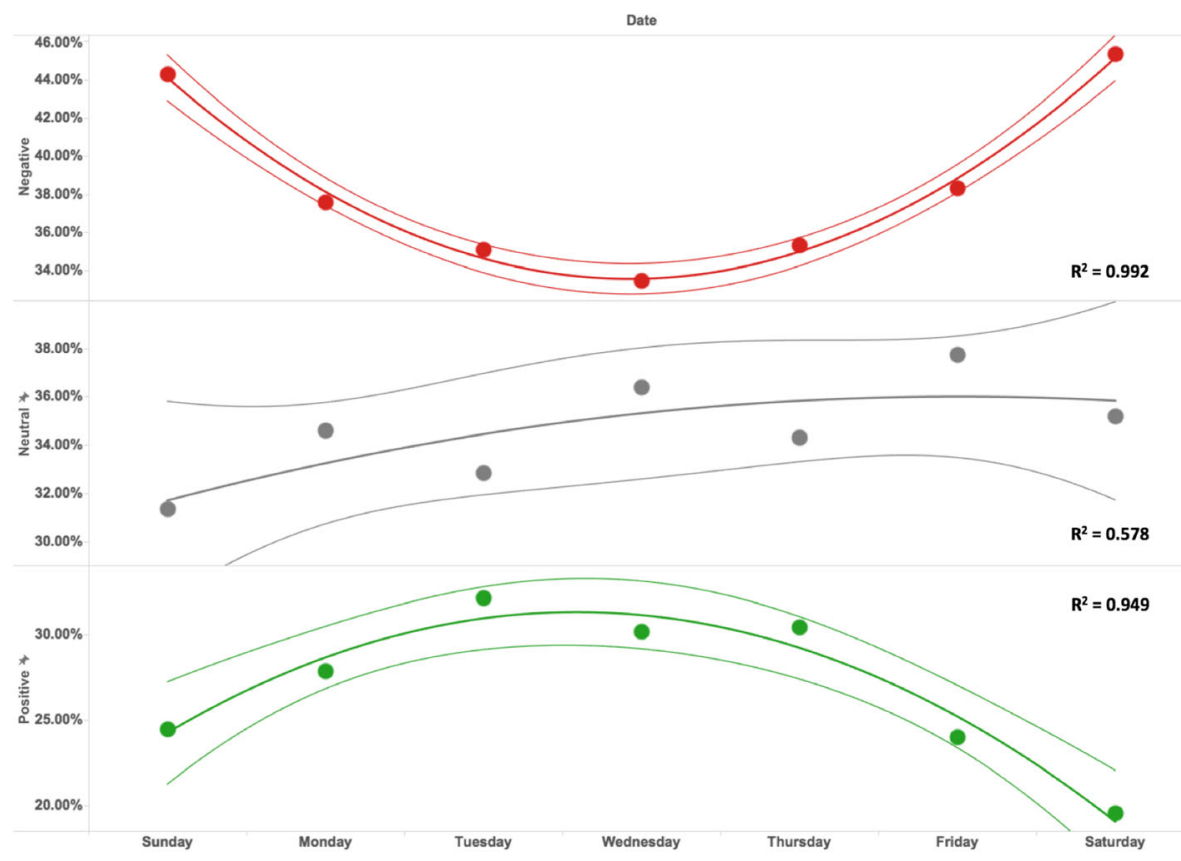

Fig. 7 The association of different days of the week with the relative proportions of tweets containing Negative, Neutral and Positive opinions 


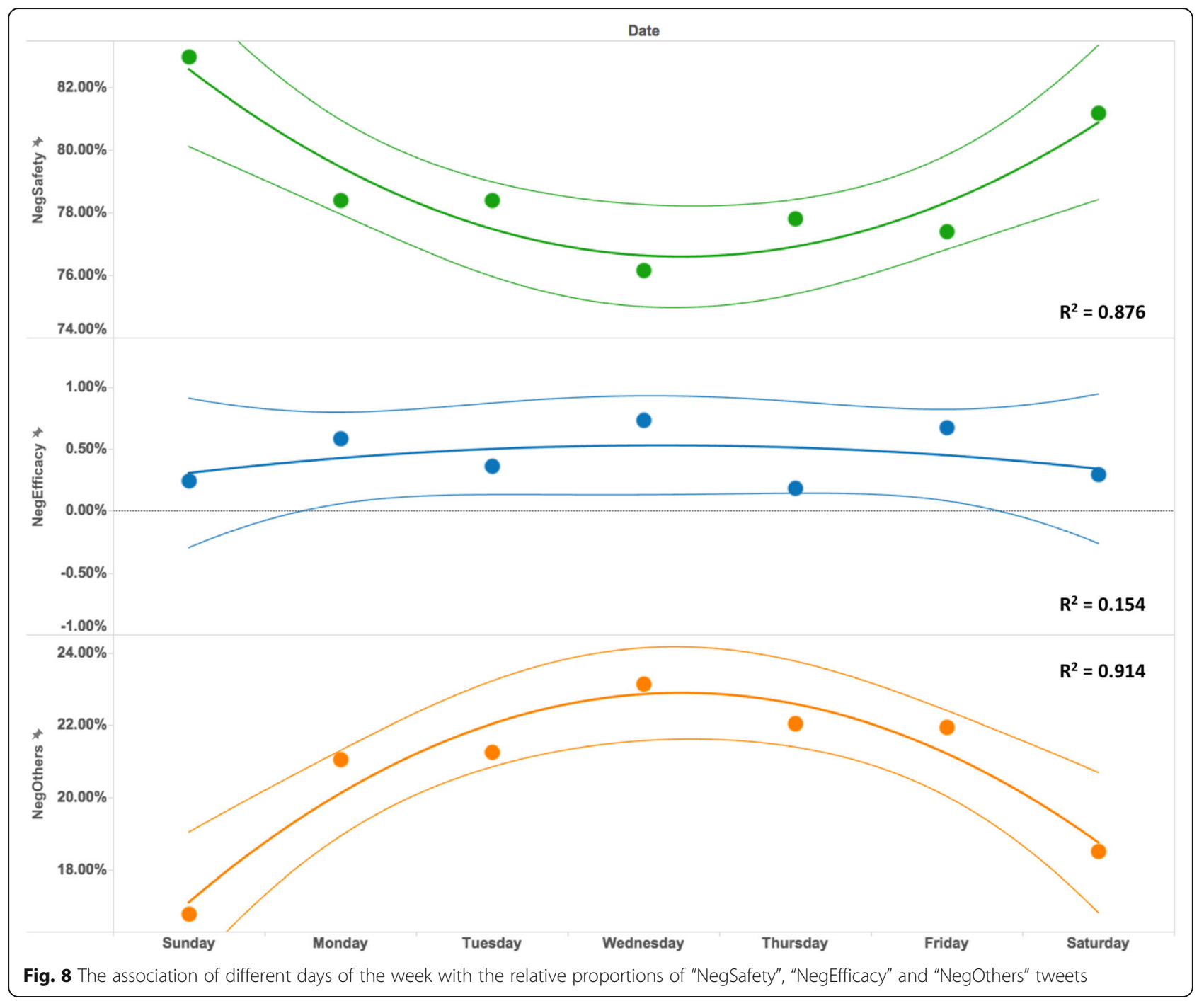

for all the tweets). It is safe to say that Twitter is not an isolated world and that promotion efforts in mainstream media could have a positive impact on public opinion on Twitter.

Further analysis helped us find more trends and patterns for different sentiments on HPV vaccines. We observed a weak trend for "Negative" tweets that decreased firstly and began to increase later; an opposite trend was identified for "Positive" tweets. We also found the tweets that contain the concerns about efficacy for HPV vaccines showed a relative significant decreasing trend. Strong associations were found between different sentiments and different days of a week. Average rates for the "Negative" tweets reached to the bottom on Wednesdays and reached the peak on weekends; an opposite association was found for "Positive" tweets. For the sub-categories of the "Negative" sentiment group, strong associations between the numbers of tweets and days of a week were also found for "NegSafety" and "NegOthers".
One third of parents are distrustful of newer vaccines [11]. Our efforts on sentiment analysis for newly approved HPV vaccines provide an automatic and instant way to extract public opinion and understand the concerns using Twitter data. Our system can provide feedback to public health professionals to monitor online public response and examine the effectiveness of their HPV vaccine promotion strategies. The associations found for different sentiments with different days of week could also be very helpful for public health professionals in adjusting their promotion plans. For example, they can deliver more persuasive messages on weekends instead of the middle of the week, as the negative opinions are more prevalent on these days.

A significant issue for the machine learning system is the un-satisfactory performance on the minority categories. However, as we observed that the proportion of those tweets is very small, this will not significantly influence the results. Another limitation of our approach is study population bias. As the Twitter population is not 
representative of the general population, the public opinions on Twitter cannot be used to fully represent the opinions of the general public. However, previous study showed the correlation between sentiments expressed online and $\mathrm{CDC}$-estimated vaccination rates [6], and therefore we believe our results are meaningful and can be used to be assess general public opinion.

\section{Conclusion}

In order to understand public opinion about HPV vaccines, we leveraged the machine learning-based sentiment analysis system to automatically extract public opinion towards HPV vaccine from a large unannotated tweets corpus that contains HPV vaccines related keywords. The evaluation of the system on the large tweets corpus was promising, with micro-averaged score at 0.786 and macro-averaged score at 0.7081 respectively on the sampling dataset. Further time series analysis was done and identified trends and patterns of different sentiments and their association with different days of the week. Our findings can be provided to the health professionals to propose more precise and efficient plan to resolve public concerns on Twitter and come up with promotion plan to increase HPV vaccine uptake finally.

\section{Additional files}

Additional file 1: Table S1. Detailed definitions of different sentiment categories for HPV vaccine related tweets. (DOC $30 \mathrm{~kb}$ )

Additional file 2: Table S2. Sample tweets predicated by the machine learning system. URLs and Twitter user names have been removed. (DOC $30 \mathrm{~kb}$ )

\section{Acknowledgments}

Not applicable.

\section{Funding}

This research and this article's publication costs were supported by the National Library of Medicine of the National Institutes of Health under Award Number R01LM011829, the National Institute Of Allergy And Infectious Diseases of the National Institutes of Health under Award Number R01Al130460, and the UTHealth Innovation for Cancer Prevention Research Training Program Pre-doctoral Fellowship (Cancer Prevention and Research Institute of Texas grant \# RP160015).

\section{Availability of data and materials}

The datasets generated during and/or analyzed during the current study are not publicly available due to time issues, but are available from the corresponding author upon reasonable request.

\section{Authors' contributions}

JD collected that data, wrote the initial draft and revised subsequent draft. JD, JX and HS developed the method, preformed the evaluation, and conducted analysis of the results. CT provided institutional support, contributed to research design, and guided the data analysis. All authors read and approved the final manuscript.

\section{Competing interests}

The authors declare that they have no competing interests.
Consent for publication

Not applicable.

\section{Ethics approval and consent to participate}

This study received IRB approval from Committee for the Protection of Human Subjects at The University of Texas Health Science Center at Houston. The reference number is HSC-SBMI-16-0291.

\section{About this supplement}

This article has been published as part of BMC Medical Informatics and Decision Making Volume 17 Supplement 2, 2017: Selected articles from the International Conference on Intelligent Biology and Medicine (ICIBM) 2016: medical informatics and decision making. The full contents of the supplement are available online at https://bmcmedinformdecismak.biomedcentral.com/articles/ supplements/volume-17-supplement-2.

\section{Publisher's Note}

Springer Nature remains neutral with regard to jurisdictional claims in published maps and institutional affiliations.

Published: 5 July 2017

\section{References}

1. Chunara R, Andrews JR, Brownstein JS. Social and news media enable estimation of epidemiological patterns early in the 2010 Haitian cholera outbreak. Am J Trop Med Hyg. 2012;86:39-45.

2. Culotta A. Detecting influenza outbreaks by analyzing Twitter messages. arXiv Prepr. arXiv1007.4748. 2010;

3. Chew C, Eysenbach G. Pandemics in the age of Twitter: content analysis of Tweets during the 2009 H1N1 outbreak. PLoS One. 2010;5(Public Library of Science):e14118.

4. Freifeld CC, Brownstein JS, Menone CM, Bao W, Filice R, Kass-Hout T, et al. Digital drug safety surveillance: Monitoring pharmaceutical products in Twitter. Drug Saf. 2014;37:343-50.

5. Zhou X, Coiera E, Tsafnat G, Arachi D, Ong M-S, Dunn AG. Using social connection information to improve opinion mining: Identifying negative sentiment about HPV vaccines on Twitter. Stud Health Technol Inform. 2015;216:761-5. Netherlands.

6. Salathé M, Khandelwal S. Assessing vaccination sentiments with online social media: implications for infectious disease dynamics and control. PLoS Comput Biol. 2011;7(10):e1002199.

7. Twitter Statistics for 2016 [Internet]. Available from: https://www. brandwatch.com/2016/05/44-twitter-stats-2016/.

8. Kontopoulos E, Berberidis C, Dergiades T, Bassiliades N. Ontology-based sentiment analysis of twitter posts. Expert Syst Appl. 2013;40:4065-74. Elsevier.

9. Dunn AG, Leask J, Zhou X, Mandl KD, Coiera E. Associations between exposure to and expression of negative opinions about human papillomavirus vaccines on social media: an observational study. J Med Internet Res. 2015;17(6).

10. The HPV Vaccine: Access and Use in the U.S. [Internet]. Available from: http:// kff.org/womens-health-policy/fact-sheet/the-hpv-vaccine-access-and-use-in/.

11. Leask J. Target the fence-sitters. Nature. 2011;473:443-5. Nature Publishing Group.

12. Du J, Xu J, Song H, Liu X and Tao C. Optimization on Machine Learning Based Approaches for Sentiment Analysis on HPV Vaccines Related Tweets. J Biomed Semantics. 2017:8(1):9.

13. An In-Depth Look at the Science of Twitter Timing [Internet]. Available from: https://blog.kissmetrics.com/the-science-of-twitter-timing/.

14. The Best (And Worst) Times To Post On Social Media (Infographic) [Internet]. Available from: https://www.fastcompany.com/3036184/how-to-be-asuccess-at-everything/the-best-and-worst-times-to-post-on-social-mediainfograph.

15. HPV Sharply Reduced in Teenage Girls Following Vaccine, Study Says [Internet]. Available from: http://www.nytimes.com/2016/02/22/health/ vaccine-has-sharply-reduced-hpv-in-teenage-girls-study-says.html?_r=0. 